\title{
Intolerance of uncertainty and novelty facilitated extinction: the impact of reinforcement schedule
}

\author{
Article \\ Published Version \\ Creative Commons: Attribution 4.0 (CC-BY) \\ Open Access
}

Wake, S., Dodd, H. ORCID: https://orcid.org/0000-0003-14465338 and Morriss, J. (2021) Intolerance of uncertainty and novelty facilitated extinction: the impact of reinforcement schedule. British Journal of Psychology. ISSN 0007-1269 doi: https://doi.org/10.1111/bjop.12538 Available at https://centaur.reading.ac.uk/100922/

It is advisable to refer to the publisher's version if you intend to cite from the work. See Guidance on citing.

To link to this article DOI: http://dx.doi.org/10.1111/bjop.12538

Publisher: British Psychological Society

All outputs in CentAUR are protected by Intellectual Property Rights law, including copyright law. Copyright and IPR is retained by the creators or other copyright holders. Terms and conditions for use of this material are defined in the End User Agreement.

\section{www.reading.ac.uk/centaur}

\section{CentAUR}


Central Archive at the University of Reading

Reading's research outputs online 


\title{
Intolerance of uncertainty and novelty facilitated extinction: The impact of reinforcement schedule
}

\author{
Shannon Wake | Helen Dodd | Jayne Morriss ([)
}

Centre for Integrative Neuroscience and Neurodynamics, School of Psychology and Clinical Language Sciences, University of Reading, Reading, UK

\section{Correspondence}

Jayne Morriss, Centre for Integrative

Neuroscience and Neurodynamics, School of Psychology and Clinical Language Sciences, University of Reading, Reading, UK.

Email: j.e.morriss@reading.ac.uk

\begin{abstract}
Individuals who score high in intolerance of uncertainty (IU) display reduced threat extinction. Recently, it was shown that replacing threat associations with novel associations during extinction learning (i.e., presenting a novel tone $100 \%$ of the time) can promote threat extinction retention in individuals with high IU. This novelty facilitated extinction (NFE) effect could be driven by the tone's novelty or reliability. Here, we sought to address this question by adjusting the reliability of the novel tone (i.e., the reinforcement rate) during NFE. We measured skin conductance response during an associative learning task in which participants $(n=92)$ were assigned to one of three experimental groups: standard extinction, NFE 100\% reinforcement, or NFE 50\% reinforcement. For standard extinction, compared to NFE $100 \%$ and 50\% reinforcement groups, we observed a trend for greater recovery of the conditioned response during extinction retention. Individuals with high IU relative to low IU in the standard extinction group demonstrated a larger recovery of the conditioned response during extinction retention. These findings tentatively suggest that NFE effects are driven by the novelty rather than the reliability of the new stimulus. The implications of these findings for translational and clinical research in anxiety disorder pathology are discussed.
\end{abstract}

\section{K E Y W O R D S}

extinction, extinction retention, intolerance of uncertainty, skin conductance, threat acquisition 


\section{INTRODUCTION}

The ability to discriminate between threat and safety is essential for survival. Learning to associate potentially dangerous cues with threat can protect us from harmful situations. However, when a cue ceases to predict threat, it is adaptive to update this association from threat to safety. This process is known as threat extinction (Milad \& Quirk, 2012). During threat extinction, omission of an aversive event results in a reduction of defensive behaviours, such as sympathetic arousal and freezing. This reduction in responsivity to the learned threat cue over time is believed to reflect changes in contingency beliefs and harm expectancy (Hofmann, 2008). However, changes in contingency may not always be obvious; it may take several experiences to identify that a stimulus that once signalled threat may now signal safety. Uncertainty about changes in contingency from threat to safety might therefore prolong, or prevent, the learning of new safety associations (Bouton, 2002).

Uncertainty has been identified as an important facet of anxiety and stress disorders more broadly (Carleton, 2016a, 2016b; Dugas et al., 2004; Grupe \& Nitschke, 2013). Intolerance of uncertainty (IU) refers to individual differences in the tendency to find uncertainty aversive and has been only recently examined in relation to threat extinction learning (Morriss et al., 2015, 2016a, 2016b) and retention (Dunsmoor et al., 2015; Lucas et al., 2018; Morriss et al., 2020; Wake et al., 2021). This work has demonstrated that higher IU is associated with reduced threat extinction learning and retention, indexed by greater skin conductance responding to cues that no longer signal threat (Dunsmoor et al., 2015; Lucas et al., 2018; Morriss et al., 2015, 2016a; Morriss \& van Reekum, 2019; Wake et al., 2021).

During traditional threat extinction learning and retention, the unconditioned stimulus (US) is omitted. This omission may result in the conditioned stimulus (CS) adopting one of two new meanings (Dunsmoor et al., 2015): the CS may be interpreted as an uncertain threat cue or, alternatively, as a safety cue. It is thought that the uncertainty surrounding the meaning of the CS during threat extinction is what maintains the conditioned response in individuals with high IU (Morriss \& van Reekum, 2019; Morriss et al., 2019). This explanation is in line with a current definition of IU, where the omission of information sustains the perception of uncertainty in individuals with higher IU (Carleton, 2016b, p.31).

The process of threat extinction has formed the basis of clinical interventions such as exposure therapy (Milad \& Quirk, 2012). In exposure therapy, patients are gradually exposed to the things they fear. Over multiple exposure sessions, fear of particular objects, activities, or situations is reduced. However, many patients experience a return of symptoms after exposure therapy due to treatment effects failing to remain long-term (Craske et al., 2014; Graham \& Milad, 2011). Given poorer threat extinction in individuals with higher IU, we can speculate that IU may be one of the reasons why some patients with anxiety and stress disorders relapse after exposure treatment. There is therefore a compelling incentive to identify new methods to promote threat extinction learning and retention generally and particularly in participants with high levels of IU.

Dunsmoor et al. (2015) proposed a novelty facilitated extinction (NFE) procedure to aid threat extinction learning and retention. In NFE, a surprising stimulus such as a novel tone is presented with the CS+, rather than nothing as in standard extinction. Dunsmoor et al. (2015) suggests that the novel tone creates a bigger prediction error, which forms a stronger extinction memory compared to the mere omission of the US. To examine this hypothesis, Dunsmoor and colleagues used a differential threat conditioning paradigm to train participants to associate a CS (CS+) with an aversive US. The US was paired with the CS+ during 33\% of acquisition trials. Another CS (CS-) was always presented alone. During extinction learning, one group of participants was presented with standard extinction training, while a second group was presented with a novel, non-aversive tone in 100\% of CS+ extinction trials. Findings demonstrated that the NFE group versus the standard extinction group exhibited greater threat extinction retention 24 hours later. Furthermore, self-reported IU was associated with poorer threat extinction retention following standard extinction, but not after NFE. Similar results for standard extinction and NFE have also been found after reinstatement (Lucas et al., 2018). However, another laboratory failed to replicate NFE after avoidance conditioning (Krypotos \& Engelhard, 2018), suggesting that the parameters for NFE to occur may be particularly limited. Taken together, results 
from these studies suggest that NFE is more effective than standard extinction in improving extinction retention in general and in individuals with higher IU.

Both Dunsmoor et al. (2015) and Lucas et al. (2018) employed a 100\% reinforcement schedule during NFE training. Logically, NFE with 100\% reinforcement is as reliable as standard extinction with $0 \%$ reinforcement. However, NFE with 100\% reinforcement may be perceived as a more reliable indicator of whether a shock is likely to occur because the shock is being replaced with a novel tone, while in standard extinction with $0 \%$ reinforcement, the shock is being overlaid with nothing. The lack of reinforcement in standard extinction may leave the participant guessing as to whether the shock will occur. Therefore, it is unclear as to whether NFE effects occur because of the novelty or reliability of the tone. One way to examine this question would be to address whether NFE effects hold if the novel tone is less reliable, for example, if a 50\% reinforcement schedule is used. Moreover, it will be important to examine the role of reinforcement rate in NFE generally and in relation to IU across extinction learning and retention phases.

Understanding how reinforcement schedule influences NFE will have implications for future exposure based therapies. For example, NFE may only succeed in situations with reliable reinforcement schedules between the CS and US, that is, in a controlled setting during exposure steps with a therapist. However, NFE may be unlikely to work in real-life exposures where there are more complex environments and unreliable reinforcement schedules between the CS and US, that is, social environments with animals or people who may behave unpredictably.

Here, we adjusted the reinforcement schedule during NFE to assess whether improved extinction retention after NFE training (Dunsmoor et al., 2015; Lucas et al., 2018) is driven by the reliability of the tone. Participants underwent partial threat acquisition and then one of three extinction phases: $100 \%$ reinforcement NFE group, a 50\% reinforcement NFE group, or a standard extinction training group. To ensure that an equal number of participants were assigned to each group, a second experimenter allocated participants into conditions based on their IU score. We measured skin conductance response while participants underwent threat acquisition, standard extinction learning, or NFE (100\% reinforcement or $50 \%$ reinforcement) and extinction retention. The extinction retention phase took place $24 \mathrm{~h}$ after acquisition and extinction learning. We split extinction learning and retention into early and late (i.e., first or last 8 CS+/CS - trials). Similar to Dunsmoor et al. (2015), we used (1) angry faces as the CS and electric shock as the US during acquisition, and (2) a 33\% reinforcement schedule during acquisition. A non-aversive neutral tone was used in both the $100 \%$ reinforcement NFE and $50 \%$ reinforcement NFE conditions. All participants were presented with both the CS+ and CS - without any reinforcement of shocks or tones during the extinction retention phase.

We hypothesised that during threat acquisition, skin conductance responding would be higher to learned threat $(\mathrm{CS}+)$ versus learned safety $\left(\mathrm{CS}^{-}\right)$cues, indicative of conditioned responding. With regards to the different extinction groups, we hypothesised that (1) if it is the novelty of the tone that drives NFE, reduced conditioned responding for the CS+ versus CS- should be observed during early extinction retention in both of the NFE groups; (2) if it is the reliability of the tone that drives NFE, reduced conditioned responding for the CS+ versus CS - should be observed during early extinction retention for the NFE group with 100\% reinforcement, compared to the NFE 50\% reinforcement group and standard extinction groups.

Based on previous IU research, we predicted that in the standard extinction group, higher IU, relative to lower IU, would be associated with larger conditioned responding to CS+ versus CS- cues during the late part of the extinction learning phase (Morriss et al., 2015, 2016b). Moreover, we expected that higher IU, relative to lower IU in the standard extinction group, would be associated with larger conditioned responding to CS+ versus CS- cues during the early part of the extinction retention phase (spontaneous recovery scores or the first 8 trials) (Wake et al., 2021).

If it is the reliability the new CS+ association, rather than the novelty of the new CS+ association during NFE that impacts extinction retention in high IU individuals, we should observe higher IU in the $100 \%$ reinforcement NFE group to exhibit reduced conditioned responding to CS+ versus CScues during the early part of the extinction retention phase (spontaneous recovery scores or the first 
8 trials) compared to higher IU in the 50\% reinforcement NFE group and standard extinction group. However, this hypothesis was somewhat exploratory given that no research has examined the effect of the reinforcement schedule during NFE and IU on extinction learning and retention.

In line with our previous work (for a discussion see Morriss et al., 2016), we tested the specificity of IU effects by controlling for trait anxiety, assessed by the State-Trait Inventory for Cognitive and Somatic Anxiety (STICSA) (Ree et al., 2008). We selected the STICSA because it is thought to be a purer measure of anxiety, compared to other trait anxiety measures, which also feature depressive symptomology (Grös et al., 2007).

In our original registered report, we included eyetracking. However, with agreement of the editor, we have removed the collection of eyetracking in this study because we have recently observed differences during extinction for skin conductance response (SCR) depending on whether eyetracking is present or absent. In extinction studies without eyetracking (Morriss et al., 2016, 2019), we have observed significant interactions between stimulus $\mathrm{x}$ time effects on SCR, that is, larger SCR for the CS +vs. CS - at the beginning of extinction learning and comparable SCR for the CS+ and CS - at the end of extinction learning. However, in recent preregistered studies with eyetracking, we have only observed significant effects of stimulus on SCR, and no significant interaction between stimulus $\times$ time in our control groups, suggesting that participants continue responding to the CS+ vs. CS- (Morriss et al., 2020; Wake et al., 2020). The two recent studies with eyetracking have near identical procedures to older studies without eyetracking. This leaves us to believe that the experimental paradigm in combination with the use of a head-mounted eyetracker elicits greater anxious arousal in participants. These changes were made before any data collection took place.

\section{METHOD}

\section{Participants}

Ninety-two participants were recruited to take part in this study (sex: 62 female, 30 males; $M$ age $=22.53$, $S D$ age $=4.53$; age range $=19-37$; ethnicity: 63 White, 8 Multi-Ethnic, 7 Middle-Eastern $/$ Arab, 4 Asian, 3 Black, 2 Latin American, and 5 not specified; sexual orientation: 66 heterosexual, 14 sexual minorities (lesbian/gay/bisexual/pansexual), and 12 not specified). One participant did not return for the second day of testing and so did not have Day 2 data, one participant was excluded for technical reasons, and nine participants were excluded due to being defined as non-responders (see criteria below). Therefore, 82 participants were included in the analyses of Day 1, and 81 participants were included in the analyses of Day 2.

Multilevel models (MLM) were used to analyse experimental data, where IU and STICSA scores were entered into the analysis as continuous predictor variables. MLMs can account for missing cases and therefore are more powerful than repeated measures ANOVAs (Quené \& Van den Bergh, 2004). Despite this, due to the complexity of the approach, there is no agreed upon method for calculating power and estimating sample size for MLM (Peugh, 2010; Snijder, 2005). Therefore, appropriate sample sizes were estimated based upon power analyses using a repeated measures ANCOVA. The sample size of this study is based on a power analysis using the effect size $\left(\eta_{p}^{2}=0.12\right)$ from a previous NFE experiment (CS X Group interaction from the extinction retention phase of Dunsmoor et al. (2015)). The following parameters were used: effect size $\mathrm{f}=0.37$ (converted from $\eta_{p}^{2}=0.12$ ), $\alpha$ error probability $=0.05$, Power $(1-\beta$ error probability $)=0.8$, number of groups $=3($ EXT, NFE $50 \%$, NFE 100\%), numerator $d f=2$, and number of covariates $=2$ (IU, STICSA). The total sample size suggested was $n=74$. Due to non-responding in SCR (typically $5-10 \%$ of sample), we aimed to collect 16 more participants to the total desired sample size to retain good statistical power. The total sample size aim for the current study was $n=90$.

Data collection was terminated when the required sample size was met. In the case of participant withdrawal or errors in recording (i.e., equipment failure), replacement participants were recruited. 
Participants were recruited from the University of Reading and the local area through the use of advertisements and word of mouth. We recruited participants who were between the age of 18 and 35 years and who were free of psychotropic medication. No other exclusion criteria were used for recruitment, and we did not restrict recruitment based on IU score. Participants were paid $£ 15$ to remunerate them for their time, $f_{5}$ after the first day of testing and $f_{10}$ after completing the extinction retention phase on day 2.

The procedure was reviewed and accepted by the University of Reading Research Ethics Committee.

\section{Procedure}

On the first day of the experiment, participants were informed about the experimental procedures upon arrival at the laboratory. Participants were then seated in the testing booth and asked to complete a consent form and a series of questionnaires (see below) on the computer screen. To ensure that an even number of high and low participants were assigned to each condition of the experiment, the researcher allocated each participant to a condition based on their IU score. Based on previous research, low IU is defined as <average score of 65 and high IU is defined as => 65 (Morriss \& van Reekum, 2019).

At this time, physiological sensors and the stimulator electrode were attached to the participants' left hand. An initial shock was delivered to the participant at a very low level $(0.5 \mathrm{~mA})$ and was gradually increased in steps of $0.5 \mathrm{~mA}$. After the delivery of each shock, the participant was asked to rate the sensation on a scale of 1 ('not painful at all') to 10 ('extremely painful'). When a rating of ' 8 ' was reached on this scale, the experimenter reduced the intensity of the shock by 1 step and informed the participant that the intensity of the shock would remain the same for the duration of the experiment (this procedure is based on that used by Delgado et al., 2008).

Before the task started, participants were be instructed to maintain attention to the task by looking at the faces and to stay as still as possible. Participants in all experimental groups were asked to wear headphones, which they will be told were needed to block out background noise. This is a cover story for the NFE 50\% and NFE 100\% groups who later received surprising neutral tones through the headphones during the augmented extinction phase (Dunsmoor et al., 2015). The conditioning task (see 'Conditioning Task' for details) was presented on a computer screen while skin conductance response was recorded.

On the second day ( $24 \mathrm{~h}$ later), participants arrived at the laboratory and were informed of the experimental procedures. The same computer and physiological setup were used as described above. Participants received the same instructions as administered on the first day; however, participants were not presented with any shocks or tones during the extinction retention phase. Each session took approximately 30 minutes in total.

\section{Conditioning task}

The conditioning task was designed using E-Prime 2.0 software (Psychology Software Tools Ltd, Pittsburgh, PA). Participants sat approximately $60 \mathrm{~cm}$ from the screen. Visual stimuli were angry faces (Ekman \& Friesen, 1976). The electric shock US was delivered through a MLADDF30 stimulating bar electrode attached to the participants left hand and connected to an ADInstruments Isolated Stimulator built into Powerlab 26T (AD Instruments Ltd, Chalgrove, Oxfordshire). The intensity of the shock was determined by the participants themselves prior to the start of the task through a standardised procedure described above. The maximum intensity of shock permitted to be administered was $10 \mathrm{~mA}$. The mean shock intensity across participants was $7.08 \mathrm{~mA}(S D=3.03)$, and the mean pain rating of the sensation was $6.18(S D=1.66)$ on a scale ranging from 1 ('not painful at all') to 10 ('extremely painful'). 
The task comprised three phases: acquisition, extinction learning (standard extinction or NFE), and extinction retention. Acquisition and extinction occurred on day 1 and extinction retention occurred $24 \mathrm{~h}$ later on day 2. During acquisition, one of the angry faces was paired with the electric shock $33 \%$ of the time (CS+), while the other angry face was always be presented alone (CS-). Conditioning contingencies were counterbalanced. Following acquisition, threat extinction took place during which angry faces were presented in the absence of the electric shock (US) (see Figure 1). During this phase, participants assigned to the NFE 100\% (novelty facilitated extinction) group were presented with a low-volume (60 decibel) $440 \mathrm{~Hz}$-tone with every CS+ trial in place of the electric shock (100\% paired). Participants allocated to the NFE 50\% group were presented with the tone in place of the electric shock during $50 \%$ of CS + trials (50\% paired). Participants in the EXT group were not presented with a tone during any CS+ trials. Participants returned to the laboratory 24 hours later to take part in the extinction retention phase during which the angry faces were presented to all participants in the absence of electric shock and sound stimuli.

The acquisition phase consisted of 40 trials (8 CS+ paired, $16 \mathrm{CS}+$ unpaired, $16 \mathrm{CS}-$ ). The extinction phase comprised 32 trials (16 CS+ and 16 CS-), where early was defined as the first $8 \mathrm{CS}+/ \mathrm{CS}-$ trials and late was defined as the last $8 \mathrm{CS}+\mathrm{CS}-$ trials. The extinction retention phase included 32 trials (16 CS+ unpaired and 16 CS-), where early was defined as the first $8 \mathrm{CS}+/ \mathrm{CS}-$ trials and late was defined as the last 8 CS+/CS - trials (see Figure 1). Experimental trials were pseudo-randomised such that the first trial of acquisition was always paired and then after all trial types were randomly presented. The angry faces were presented for a total of $6000 \mathrm{~ms}$. Subsequently, a blank screen was presented for 12,000$14,000 \mathrm{~ms}$. During reinforced trials, the electric shock was delivered for $200 \mathrm{~ms}$ and co-terminated with the angry face stimulus. During NFE trials, the tone was presented for $1500 \mathrm{~ms}$ and co-terminated with the trial.

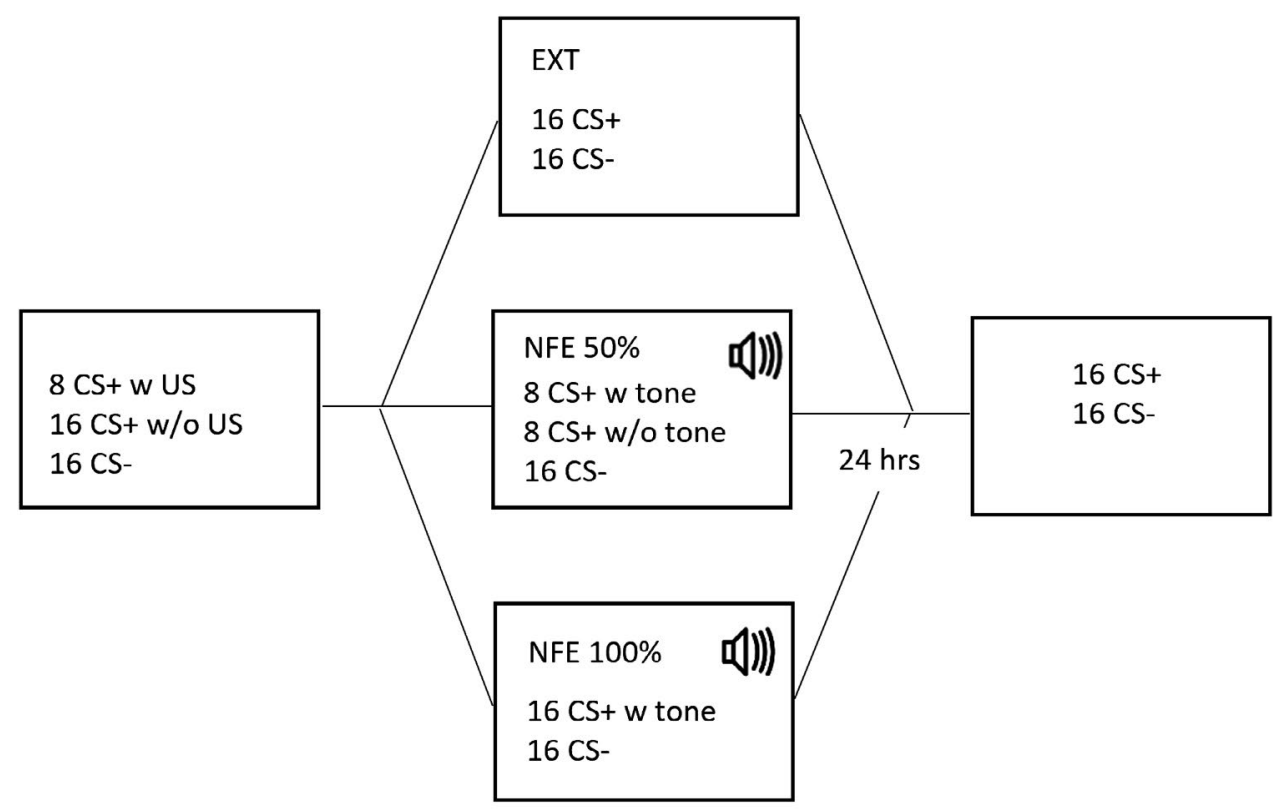

Threat Acquisition

Extinction Learning

Extinction Retention

F I G U R E 1 Image illustrating stimulus types, experimental group and procedure. Ext = Standard Extinction Group, NFE 50\% $=50 \%$ Reinforced Novelty Facilitated Extinction Group; NFE 100\% $=100 \%$ Reinforced Novelty Facilitated Extinction Group. 


\section{Questionnaires}

To assess IU and trait anxiety, we administered the Intolerance of Uncertainty Scale (Freeston et al., 1994) and STICSA questionnaires (Ree et al., 2008). The IU measure consists of 27 items that are rated on a 5 -point Likert scale. The STICSA consists of 21 items that are rated on a 4 -point Likert scale. We report the mean, standard deviation, range, and internal reliability (Cronbach Alpha) of the questionnaire measures.

\section{Skin conductance acquisition and scoring}

Physiological recordings were obtained using AD Instruments (AD Instruments Ltd, Chalgrove, Oxfordshire) hardware and software. Electrodermal activity was measured with dry MLT116F silver/ silver chloride bipolar finger electrodes that were attached to the distal phalanges of the index and middle fingers of the non-dominant hand. A low constant-voltage AC excitation of $22 \mathrm{mVrms}$ at $75 \mathrm{~Hz}$ was passed through the electrodes, which were connected to a ML116 GSR Amp, and converted to DC before being digitized and stored. A PowerLab 26T Unit Model was used to amplify the skin conductance signal, which was digitized through a 16-bit A/D converter at $1000 \mathrm{~Hz}$. The electrodermal signal was converted from volts to microSiemens using AD Instruments software (AD Instruments Ltd, Chalgrove, Oxfordshire).

SCR was marked using ADinstruments software (AD Instruments Ltd, Chalgrove, Oxfordshire) and extracted using Matlab R2017a software (The MathWorks, Inc., Natick, Massachusetts, United States). SCR was scored when there was an increase of skin conductance level exceeding 0.03 microSiemens (Dawson et al., 2000). The amplitude of each response was scored as the difference between the onset and the maximum deflection prior to the signal flattening out or decreasing. SCR onsets and respective peaks were counted if the SCR onset was within 0.5-3.5 s (CS response) following CS onset (Morriss et al., 2018). Trials with no discernible SCRs were scored as zero. SCR magnitudes were square root transformed to reduce skew and z-scored to control for individual differences in skin conductance responsiveness (Ben-Shakhar, 1985). SCR magnitudes were calculated by averaging SCR-transformed values for each condition (Acquisition CS+; Acquisition CS-; Extinction Learning CS + Early; Extinction Learning CS- Early; Extinction Learning CS+ Late; Extinction Learning CS- Late; Extinction Retention CS+ Early; Extinction Retention CS- Early; Extinction Retention CS+ Late; and Extinction Retention CS- Late). We defined non-responders as those who responded to $10 \%$ or less of all trials (Morriss et al., 2018; Xia et al., 2017). Non-responders were excluded from the SCR analyses.

\section{Analyses}

The analysis was conducted using the mixed procedure in SPSS 24.0 (SPSS, Inc; Chicago, Illinois). We conducted separate MLMs for SCR magnitude during acquisition, extinction learning, and extinction retention. For SCR magnitude during the acquisition phase, we entered Group (NFE 100\%, NFE 50\%, EXT) and Stimulus (CS+, CS-) at level 1 and individual subjects at level 2. For SCR magnitude during the extinction learning phase, we entered Group (NFE 100\%, NFE 50\%, EXT), Stimulus (CS+, CS-), and Time (Early: first 8 CS+/CS - trials, Late: last 8 CS+/CS - trials) at level 1 and individual subjects at level 2. For SCR magnitude during the extinction retention phase, we entered Group (NFE 100\%, NFE $50 \%$, EXT), Stimulus (CS+, CS-), and Time (Early: first 8 CS+/CS - trials, Late: last 8 CS+/CS - trials) at level 1 and individual subjects at level 2. We included the following individual difference predictor variables in all of the multilevel models: IU and STICSA.

In all models, we used a diagonal covariance matrix for level 1. Random effects included a random intercept for each individual subject, where a variance components covariance structure was used. Fixed 
effects include Group, Stimulus, and Time. We used a maximum likelihood estimator for the multilevel models. We corrected post-hoc tests for multiple comparisons using the Benjamini-Hochberg False Discovery Rate procedure (Benjamini \& Hochberg, 1995).

In the MLMs, a significant interaction with one predictor variable (IU, STICSA) but not the other suggests specificity. Based on our prior work, we expected such specificity for IU, but explored interactions with STICSA, given findings with trait anxiety in the conditioning literature (Lonsdorf \& Merz, 2017). Where a significant interaction was observed with IU (or STICSA), we performed follow-up pairwise comparisons on the estimated marginal means of the relevant conditions estimated at specific IU values of + or -1 SD of mean IU, adjusted for STICSA (or IU). Similar analyses have been published elsewhere (Morriss et al., 2016, 2017).

Further, we calculated a 'spontaneous recovery index' by dividing each participant's mean SCR to the first 3 CS+ trials during extinction retention by the largest SCR to the CS+ during acquisition on day 1. As suggested by Dunsmoor et al. (2015), 'this value provides a percent of recovery as a function of initial acquisition, with higher values indicating a stronger recovery as a function of initial conditioning, whereas lower values indicate lower recovery as a function of initial conditioning'. We carried out a correlation between the spontaneous recovery index and IU scores to determine whether threat recovery following extinction learning is associated with individual differences in IU.

\section{RESULTS}

\section{Questionnaires}

The self-reported anxiety measures were normally distributed and had good internal reliability (see Figure 2): IU $(M=62.40, S D=17.62$, range $=32-109, \alpha=0.93)$; STICSA $(M=39.59, S D=9.66$, range $=22-62 ; \alpha=0.89)$. IU was significantly positively correlated with STICSA, $r(82)=.67, p<.001$. The EXT, NFE 50\%, and NFE 100\% groups had a similar range of scores for the IU and STICSA questionnaires (EXT IU: $M=63.22, S D=16.95$; EXT STICSA: $M=38.67, S D=10.62$; NFE 50\% IU: $M=64.36, S D=18.40$; NFE 50\% STICSA: $M=41.18$, $S D=9.65$; NFE $100 \%$ IU: $M=59.56$, $S D=17.76$; NFE $100 \%: M=38.85, S D=8.79)$.

\section{Acquisition}

During acquisition, there was a main effect of Stimulus as SCR was significantly greater towards the CS + compared to the CS $-[F(1,82)=8.33, p<.005$; see Table 1]. There were no significant main effects of Group or interactions with STICSA or IU for SCR during acquisition, $\max F=1.89$. There was a significant Stimulus x Group interaction $[F(2,82)=3.17, p=.047]$. SCR was significantly greater towards the CS+ compared to the CS - for participants in the Ext and NFE 100\% groups (Ext: $p=.004$; NFE $50 \%: p=.021)$. Participants in the NFE 50\% group did not demonstrate differential SCR between the $\mathrm{CS}+$ and the CS- during acquisition $(p=.726)$; see Table 1.

\section{Extinction learning}

During extinction learning, no differential SCR was observed for the CS+ versus CS- and SCR did not drop across time based on stimulus type (CS+, CS - ) or generally [Stimulus, $F(1,243.26)=1.16$, $p=.28$; Time, $F(1,243.26)=0.64, p=.42$; Stimulus $\times$ Time, $F(1,243.26)=0.32, p=.57]$. In addition, no main effects or interactions with Group (Ext, NFE 100\%, NFE 50\%) were observed [Group, $F(2$, $80.60)=1.06, p=.35$; Group $x$ Stimulus $\times$ Time, $F(2,243.26)=1.84, p=.16$; see Table 1 and Figure 3]. 

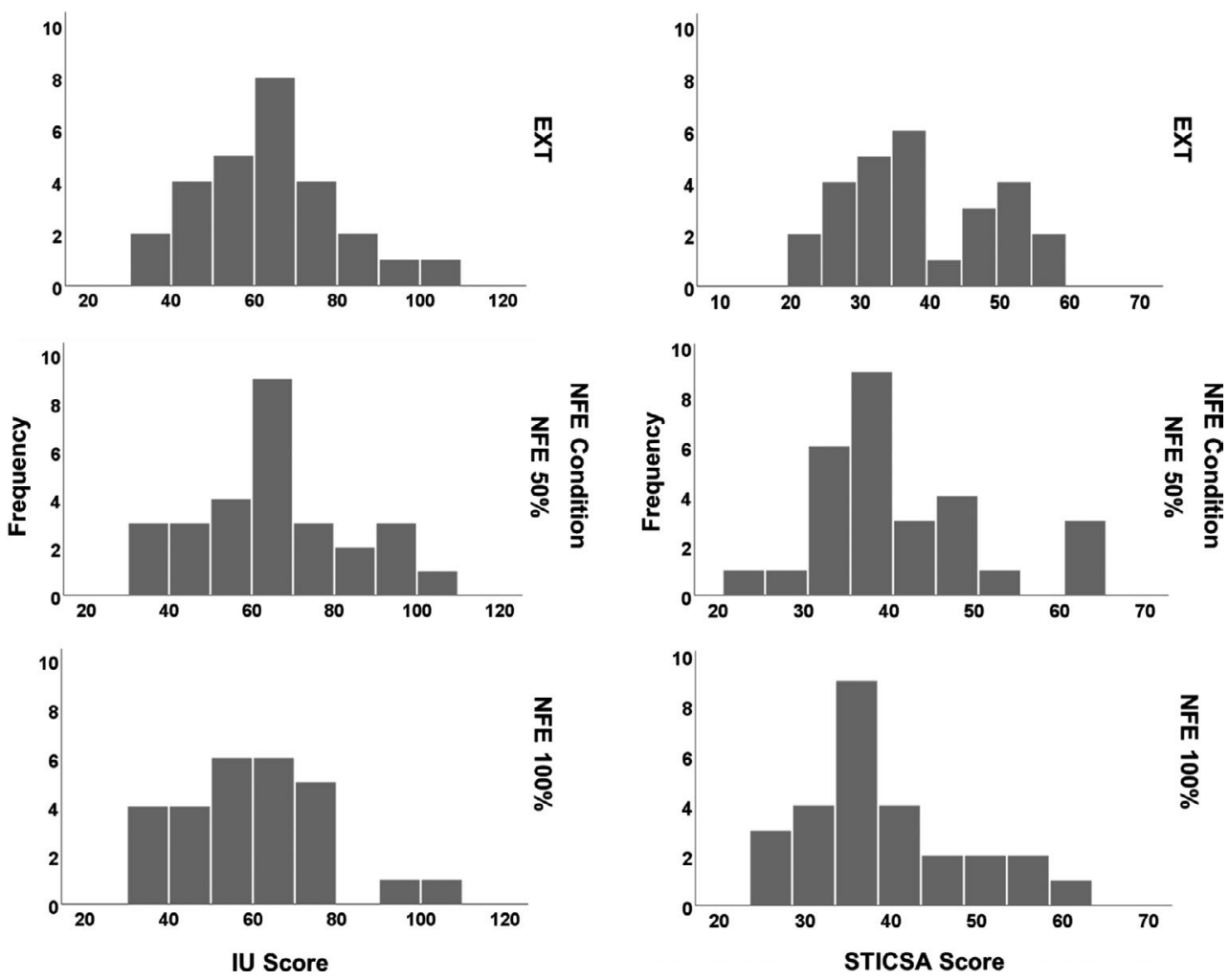

F I G U R E 2 Histograms depicting the distribution of IU and STICSA self-report scores per experimental group. Ext $=$ Standard Extinction Group, NFE 50\% = 50\% Reinforced Novelty Facilitated Extinction Group; NFE 100\% $=100 \%$ Reinforced Novelty Facilitated Extinction Group.

There were no significant interactions with IU for SCR during extinction learning, $\max F=2.66$. Individual differences in STICSA were related to SCR during extinction learning but none of the follow up pairwise comparisons survived Benjamini-Hochberg corrections (for completeness, the uncorrected results are presented in the Supplementary material).

\section{Extinction retention}

During extinction retention, there was a significant Group x Stimulus interaction $[F(2,238.54)=3.08$, $p=.048$; see Table 1, Figures 3 and 4]. Participants in the EXT group demonstrated greater SCR to the CS+ $(M=.000, S E=.059)$ versus the $\mathrm{CS}-(M=-.148, S E=.063)$ during extinction retention at trend level, $p=.054$, whereas participants in the NFE 50\% (CS+: $M=.087$, SE = .059; CS-: $M=.205$, $S E=.063)$ and NFE 100\% (CS+: $M=.042, S E=.059$; CS-: $M=-.007, S E=.063)$ groups did not demonstrate a significant differential response between the CS + and CS $-(\mathrm{NFE} \mathrm{50 \% :} p=.12$; NFE 100\%: $p=.52$ ). In addition, SCR for the CS - was lower for the EXT group, relative to the NFE $50 \%$ group, $p<.001$. No other comparisons for the CS + or CS - between groups survived Benjamini-Hochberg corrections. Further follow up t-tests demonstrated that the difference between CS+ and CS - during extinction retention significantly differed between the EXT and NFE 50\% groups $[t(52)=2.295$, $p=.026]$, but not the EXT and NFE $100 \%$ groups $[t(51)=.861, p=.393]$ or the NFE $50 \%$ and $100 \%$ groups $[t(52)=-1.517, p=.135]$. 


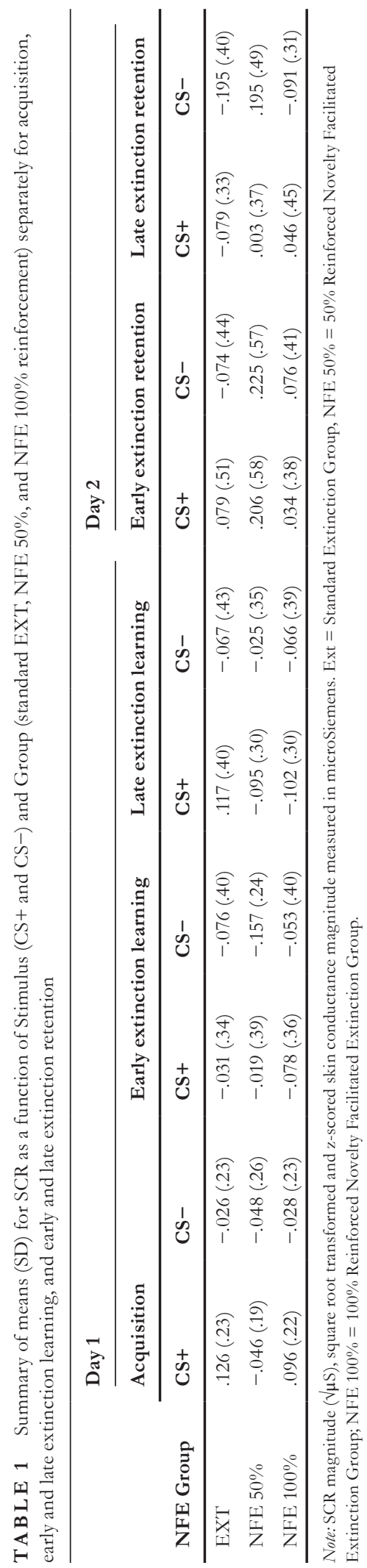




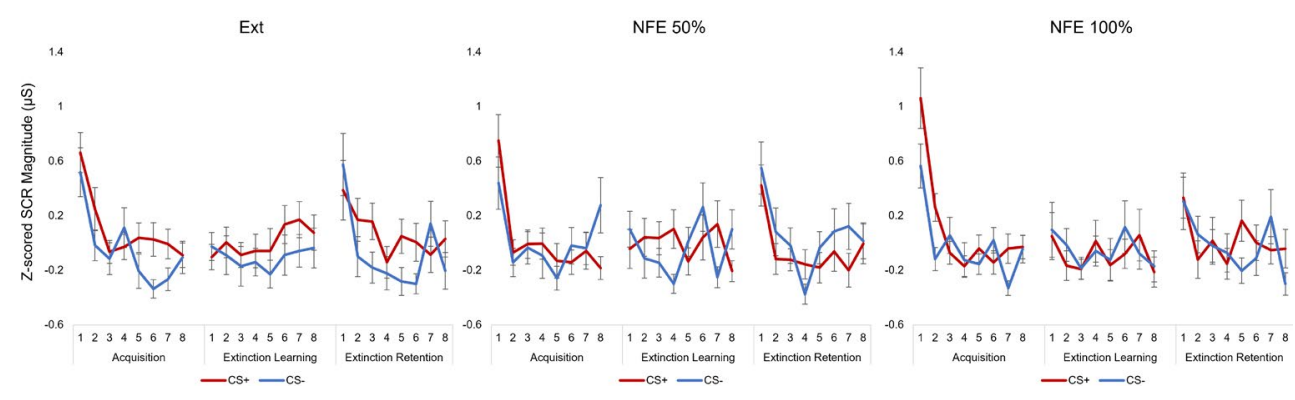

F I G U R E 3 Line graphs displaying mean SCR for each experimental group and stimulus type by block and phase. Blocks represent 3 trials for acquisition and 2 trials for extinction learning and retention. Plots are for visualisation purposes only. Error bars represent standard error. SCR magnitude $(\sqrt{ } \mu \mathrm{S})$, square root transformed and z-scored skin conductance magnitude measured in microSiemens. Ext $=$ Standard Extinction Group, NFE 50\% $=50 \%$ Reinforced Novelty Facilitated Extinction Group; NFE 100\% $=100 \%$ Reinforced Novelty Facilitated Extinction Group.

0.3

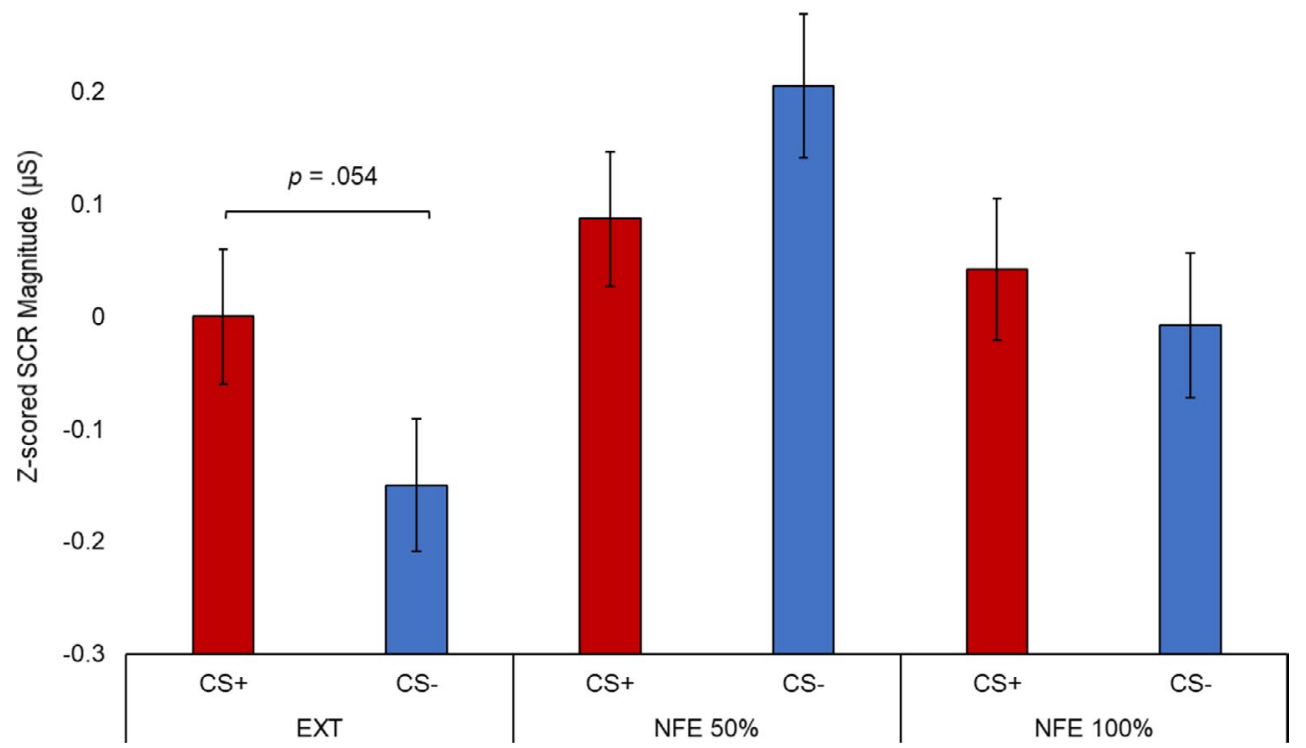

F I G U R E 4 Bar graph depicting mean SCR for each experimental group and stimulus type during extinction retention. Error bars represent standard error. SCR magnitude $(\sqrt{ } \mu \mathrm{S})$, square root transformed and z-scored skin conductance magnitude measured in microSiemens. Ext $=$ Standard Extinction Group, NFE 50\% $=50 \%$ Reinforced Novelty Facilitated Extinction Group; NFE 100\% = 100\% Reinforced Novelty Facilitated Extinction Group.

During extinction retention, SCR generally reduced across time $[F(1,238.54)=5.23, p=.02]$. In addition, the NFE 50\% group, compared to the EXT group, displayed larger SCR across extinction retention, $p=.001[F(1,78.047)=5.55, p=.006]$. No other SCR differences were observed between Groups, $p^{\prime} s>.05$. No other main effects of Stimulus $[F(1,238.54)=0.40, p=.53]$ or interactions between Group $\times$ Stimulus $\times$ Time $[F(2,228.54)=1.24, p=.29]$ were observed.

There were no significant interactions with IU for SCR during extinction retention, $\max F=2.04$. Individual differences in STICSA were related to SCR during extinction retention but none of the follow up pairwise comparisons survived Benjamini-Hochberg corrections (for completeness, the uncorrected results are presented in the Supplementary material). 


\section{Spontaneous recovery index}

A significant negative correlation between the spontaneous recovery index (i.e., SCRs to first 3 CS+ trials during extinction retention divided by the largest SCR to the CS+ during acquisition) and IU scores in the EXT group emerged $[r(25)=-.458, p=.016$; see Figure $5 \mathrm{a}]$. There were no significant correlations between the spontaneous recovery index and IU scores in the NFE 50\% group $[r(25)=.208$, $p=.299]$, or in the NFE $100 \%$ group $[r(25)=.079, p=.697]$. The correlation for the spontaneous recovery index and IU in the EXT group was significantly different than the NFE 50\% [ $z=-2.45, p=.014]$ and $100 \%[z=-1.99, p=.046]$ groups. Correlations for the spontaneous recovery index and IU in the NFE $50 \%$ and $100 \%$ groups did not significantly differ from each other $[z=.046, p=.64]$.

\section{Post-Hoc exploratory analyses}

As the significant correlation between the spontaneous recovery index and IU scores within the EXT group was in the opposite direction to that expected, we conducted a further post-hoc curve estimation analysis to examine the nature of the relationship (i.e., linear, quadratic). We identified that the relationship between the spontaneous recovery index and IU scores in the EXT group was better explained (i.e.,

(a)
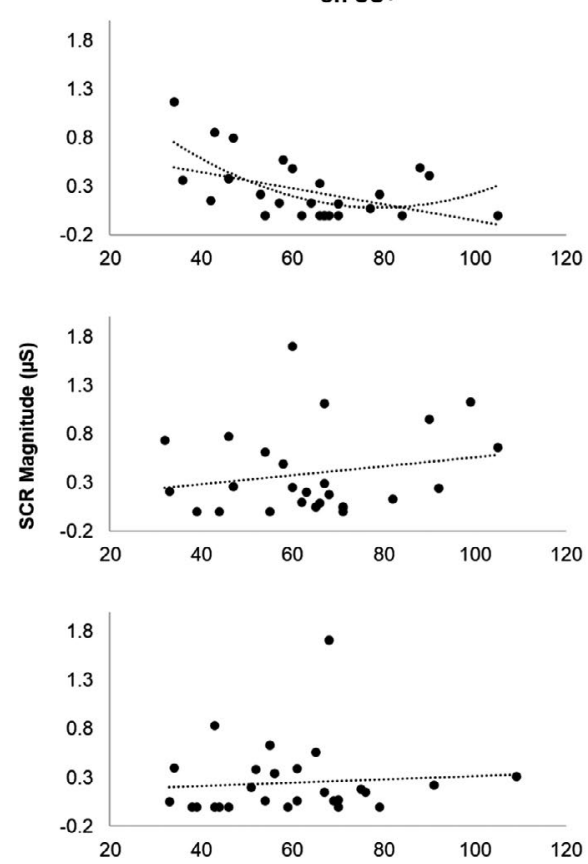

(b) Spontaneous Recovery Index based on differential CS+ versus CS-
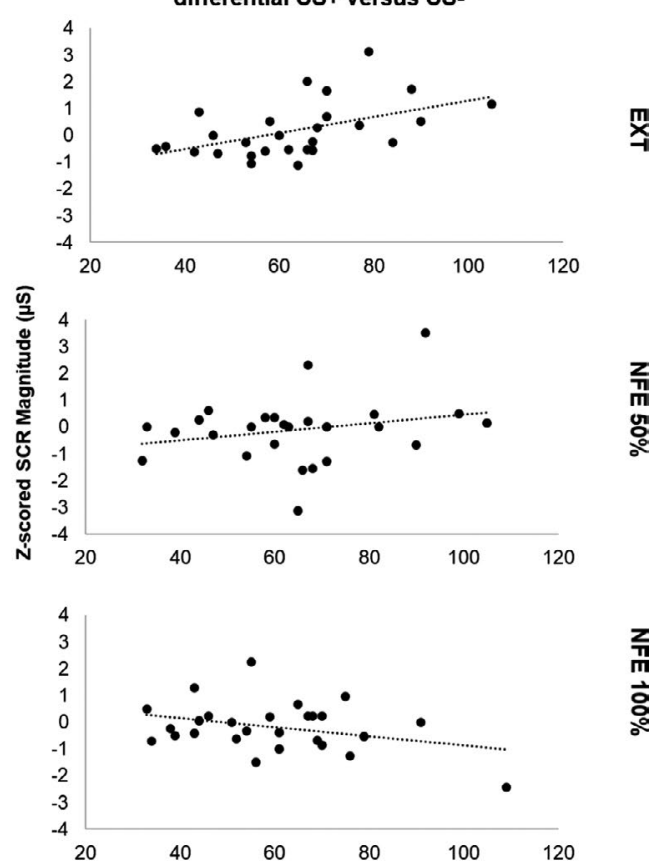

Intolerance of Uncertainty

F I G U R E 5 Correlations between the spontaneous recovery indices and IU by experimental group. Greater spontaneous recovery indexed by the first three CS + trials in extinction retention divided by the largest CS + trial in acquisition was associated with both lower and higher IU in the standard extinction group (a). Larger spontaneous recovery indexed by the first three CS+ versus CS - trials in extinction retention was associated with higher IU in the standard extinction group (b). Different metrics were used in line with the original study by Dunsmoor et al. (2015) (not z-scored) and the current study (z-scored). SCR magnitude in $(A)(\sqrt{\mu S})$, square root transformed skin conductance magnitude measured in microSiemens. SCR magnitude in $(B)(\sqrt{ } \mu \mathrm{S})$, square root transformed and z-scored skin conductance magnitude measured in microSiemens. Ext $=$ Standard Extinction Group, NFE 50\% = 50\% Reinforced Novelty Facilitated Extinction Group; NFE $100 \%=100 \%$ Reinforced Novelty Facilitated Extinction Group. 
based on the $R^{2}$ and $p$ value) by a quadratic trend $\left[R^{2}=.373, F(2,24)=7.132, p=.004\right]$, rather than a linear trend $\left[R^{2}=.209, F(1,25)=6.622, p=.016\right]$, demonstrating that both individuals with lower and higher levels of IU showed greater spontaneous recovery of the conditioned response.

As the spontaneous recovery index can also be commonly operationalised using a differential response (Lonsdorf et al., 2019), we conducted a post-hoc correlational analysis with IU and a spontaneous recovery index based on the differential response between the first three CS+ and CS - trials in extinction retention (Morriss et al., 2020; Wake et al., 2021). We found a significant positive correlation between the differential response in extinction retention and IU scores in the EXT group $[r(25)=.496$, $p=.009$; see Figure 5b] but not in the NFE 50\% group $[r(25)=.244, p=.221]$ or the NFE 100\% group $[r(25)=-.333, p=.089]$. The correlation for the differential response in extinction retention and IU in the EXT group was significantly different than the NFE $100 \%$ group $[z=3.08, p<.001]$, but not the NFE $50 \%$ group $[z=1.02, p=.3]$. Correlations for the differential response in extinction retention and IU in NFE $50 \%$ and $100 \%$ groups significantly differed from each other, $[z=2.06, p=.039$ ]. Moreover, a post-hoc curve estimation analysis confirmed that the relationship between IU and the differential response in extinction retention for the EXT group was better explained (i.e., based on the $\mathrm{R}^{2}$ and $p$ value) by a linear trend $\left[R^{2}=.246, F(1,25)=8.143, p=.009\right]$, rather than a quadratic trend $\left[R^{2}=.248\right.$, $F(2,24)=3.959, p=.033]$.

\section{DISCUSSION}

In the current study, we examined the effect of standard extinction learning and NFE reinforcement rate $(100 \%, 50 \%)$ upon extinction retention broadly and in relation to individual differences in IU and STICSA. We partially replicated and extended previous research, as we found that the standard extinction learning group exhibited a trend for a greater recovery of the conditioned response towards the CS+ relative to the CS- during extinction retention. In addition, we found partial evidence that individual differences in IU are associated with the spontaneous recovery of the conditioned response towards the CS+ following standard extinction learning, but not NFE $100 \%$ or $50 \%$ reinforcement. However, the exact relationship between IU and the spontaneous recovery of the conditioned response during extinction retention varied depending on the spontaneous recovery metric used. Finally, we failed to replicate prior IU-related patterns of compromised threat extinction in the standard extinction learning group. These findings further our understanding of how standard extinction learning and the NFE reinforcement schedule influences extinction retention generally, and in relation to IU, and has implications for future exposure-based therapies.

A typical pattern of conditioning was observed for the acquisition phase, denoted by a larger SCR to the CS+ versus CS-, for the standard extinction and NFE 100\% groups but not the NFE 50\% group. Extinction learning was rapid across all experimental groups on day one, indexed by no differential SCR to the CS+ versus CS-. For extinction retention on day two, the standard extinction learning group exhibited a larger SCR to the CS+ compared the CS- at trend, while the NFE 100\% and 50\% groups displayed no difference in SCR to the CS+ compared to the CS-. The difference between the CS+ and CS- during extinction retention for the standard extinction learning group did not significantly differ to the NFE $100 \%$ group but did to the NFE 50\% group. While we failed to directly replicate prior research (Dunsmoor et al., 2015, 2019; Lucas et al., 2018), the pattern of results are in the same direction and extend our understanding of how NFE operates under different reinforcement schedules $(100 \%$, $50 \%$ ). Because both NFE $100 \%$ and 50\% reinforcement groups showed reduced recovery of the conditioned response, we can tentatively suggest that NFE is driven by the novelty of the stimulus rather than the reliability of the stimulus presented during extinction learning.

Unexpectedly, both lower and higher IU was associated with greater spontaneous recovery of the conditioned response following standard extinction learning, but not NFE $100 \%$ or $50 \%$ reinforcement. This finding is partially in line with previous research demonstrating that higher IU is related to greater spontaneous recovery of the conditioned response after standard extinction learning, but not 
NFE 100\% reinforcement (Dunsmoor et al., 2015). These findings were based on the spontaneous recovery index used in the Dunsmoor et al. (2015) study, where the first three CS+ trials in extinction retention are divided by the largest CS + trial in acquisition. Given the debate surrounding how to operationalize the spontaneous recovery index (Lonsdorf et al., 2019), we conducted a post-hoc analysis with IU and a spontaneous recovery index based on the differential response between the first three CS+ and CS - trials in extinction retention (Morriss et al., 2020; Wake et al., 2021). With this analysis, only higher IU in the standard extinction learning group was associated with greater spontaneous recovery of the conditioned response. Interestingly, the relationship between IU and spontaneous recovery of the conditioned response was only different between the EXT and NFE 100\% groups, and not the $50 \%$ groups. These results suggest that further testing of NFE under partial reinforcement and IU is required to form firmer conclusions.

Contrary to hypotheses, we did not replicate findings of previous research that has found that higher IU, relative to lower IU, was associated with larger conditioned responding towards CS+ versus CScues during the late part of standard extinction learning (Morriss et al., 2015, 2016a, 2019; Morriss \& van Reekum, 2019). Instead, we found that the STICSA measure was related to threat extinction learning, although these results did not reach statistical significance after corrections for multiple comparisons (see Supplementary Material). Prior extinction learning research has shown specificity of IU over self-reported trait anxiety, social anxiety and worry (Morriss et al., 2016; Morriss \& van Reekum, 2019; Wake et al., 2021). However, recent published research suggests that STICSA may be more closely related to extinction-learning compared to other self-report measures of anxiety and worry (Morriss et al., 2020; Wake et al., 2020). Future work should continue to include and examine the impact of IU and STICSA upon threat extinction to disentangle shared and specific processes related to these transdiagnostic measures of anxiety (Lonsdorf \& Merz, 2017).

The findings from the present study have promising implications for translational and clinical research aiming to expand and develop new exposure based therapies for anxiety-related pathology (Craske et al., 2014; Hofmann, 2008; Milad \& Quirk, 2012). The results from this study tentatively suggest that NFE may operate similarly under reliable and unreliable environments. This will likely serve interventions like exposure-based therapy well, given that real world exposures are often littered with unavoidable uncertainty and complexity. Further research is required, however, to understand the generalisability and transferability of NFE to exposure based therapy for different clinical populations. For instance, is NFE applicable when using different types of stimuli that are relevant for a specific disorder? (i.e., trauma relevant stimuli in post-traumatic stress disorder, interoceptive stimuli for panic disorder, and food-based stimuli in eating disorders).

The study reported here had several strengths including the use of preregistered hypotheses, sample size estimates and analyses, as well as the replication and extension of the NFE experimental design. The study had a few weaknesses that can be addressed in future research. First, the conditioned and unconditioned stimuli used in the experiment could be further optimised. The use of angry male white faces as conditioned stimuli is not ideal, given that some participants may find these faces more aversive than others based on their gender and ethnic identity (Molapour et al., 2015; Olsson et al., 2005). The use of non-face stimuli as conditioned stimuli would be more appropriate to limit additional variability in responding due to these factors. Second, the use of mild electric shock as unconditioned stimuli, while popular in conditioning research (Lonsdorf et al., 2017), may induce greater variability in responding across participants because some participants receive more or less exposure to the shock during the work up procedure (i.e., depending on the shock intensity level chosen, some participants may receive 1 or 2 shocks, while some participants may receive up to 10 shocks). Alternative unconditioned stimuli such as auditory probes may be more appropriate as the auditory probe is traditionally set at the same level for all participants. These aspects may explain the lack of differential conditioning on skin conductance response in the NFE 50\% group. Third, the NFE effect in the present study and previous research (Dunsmoor et al., 2015; Lucas et al., 2018) is based on cross modal stimulus replacement (i.e., electric shock replaced with novel sound). Future research should address whether similar NFE effects can be observed under same modal 
replacement (i.e., aversive sound replaced with novel sound) and different cross modal replacement (i.e., aversive picture replaced with novel sound). Finally, the current experiment was run between two Covid-19 lockdowns in the UK and it is unknown as to what effect this would have had upon the participants taking part (i.e., having to wash their hands free of sanitiser and wear masks during the experiment).

In conclusion, the results of this experiment provide insight into how reinforcement schedule during NFE effects extinction learning and retention processes generally and in relation to individual differences in IU. The findings provide preliminary support for the use of reliable and unreliable reinforcement during NFE to override threat associations with novel associations for individuals with high IU. The results from this experiment highlight further opportunities for examining the suitability of NFE in therapies for anxiety disorder pathology that rely on threat extinction learning and retention processes, such as exposure therapy.

\section{ACKNOWLEDGEMENTS}

This research was supported by a NARSAD Young Investigator Grant from the Brain \& Behavior Research Foundation (27567) and an ESRC New Investigator Grant (ES/R01145/1) awarded to Jayne Morriss. The authors would like to thank Charlotte Elizabeth, Amanpreet Sandhu, Hritika Shah, Claudia Rodriguez-Sobstel, and Aimee Westbrook for their help in data collection.

\section{AUTHOR CONTRIBUTION}

Jayne Morriss: Conceptualization; Data curation; Formal analysis; Investigation; Methodology; Supervision; Visualization; Writing - original draft; Writing - review \& editing. Helen Dodd Conceptualization; Supervision; Writing - original draft; Writing - review \& editing. Shannon Wake: Conceptualization; Data curation; Methodology; Visualization; Writing - original draft; Writing review \& editing.

\section{DATA AVAILABILITY STATEMENT}

The materials, data, and data analyses are available on the Open Science Framework: https://osf. io/6ka8x/

\section{CONFLICTS OF INTEREST}

The authors declare no conflict of interest.

\section{ORCID}

Jayne Morriss (D) https://orcid.org/0000-0002-7928-9673

\section{REFERENCES}

Benjamini, Y., \& Hochberg, Y. (1995). Controlling the false discovery rate: A practical and powerful approach to multiple testing. Journal of the Royal Statistical Society: Series B (Methodological), 57(1), 289-300. https://doi.org/10.1111/j.2517-6161.1995. tb02031.x

Ben-Shakhar, G. (1985). Standardization within individuals: A simple method to neutralize individual differences in skin conductance. Psychophysiology, 22(3), 292-299. https://doi.org/10.1111/j.1469-8986.1985.tb01603.x

Bouton, M. E. (2002). Context, ambiguity, and unlearning: Sources of relapse after behavioral extinction. Biological Psychiatry, 52(10), 976-986. https://doi.org/10.1016/S0006-3223(02)01546-9

Carleton, R. N. (2016a). Fear of the unknown: One fear to rule them all? Journal of Anxiety Disorders, 41, 5-21. https://doi. org/10.1016/j.janxdis.2016.03.011

Carleton, R. N. (2016b). Into the unknown: A review and synthesis of contemporary models involving uncertainty. Journal of Anxiety Disorders, 39, 30-43. https://doi.org/10.1016/j.janxdis.2016.02.007

Craske, M. G., Treanor, M., Conway, C. C., Zbozinek, T., \& Vervliet, B. (2014). Maximizing exposure therapy: An inhibitory learning approach. Behaviour Research and Therapy, 58, 10-23. https://doi.org/10.1016/j.brat.2014.04.006

Dawson, M. E., Schell, A. M., \& Filion, D. L. (2000). The electrodermal system. In J. T. Cacioppo, L. G. Tassinary, \& G. G. Berntson (Eds.), Handbook of physiology, 2nd ed. (pp. 200-223). Cambridge University Press. 
Delgado, M. R., Nearing, K. I., Ledoux, J. E., \& Phelps, E. A. (2008). Neural circuitry underlying the regulation of conditioned fear and its relation to extinction. Neuron, 59(5), 829-838. https://doi.org/10.1016/j.neuron.2008.06.029

Dugas, M. J., Buhr, K., \& Ladouceur, R. (2004). The role of intolerance of uncertainty in etiology and maintenance of generalized anxiety disorder. In R. G. Heimberg, C. L. Turk, \& D. S. Mennin (Eds.), Generalized anxiety disorder: Advances in research and practice (pp. 143-163). Guilford Press.

Dunsmoor, J. E., Campese, V. D., Ceceli, A. O., LeDoux, J. E., \& Phelps, E. A. (2015). Novelty-facilitated extinction: Providing a novel outcome in place of an expected threat diminishes recovery of defensive responses. Biological Psychiatry, 78(3), 203209. https://doi.org/10.1016/j.biopsych.2014.12.008

Dunsmoor, J. E., Kroes, M. C., Li, J., Daw, N. D., Simpson, H. B., \& Phelps, E. A. (2019). Role of human ventromedial prefrontal cortex in learning and recall of enhanced extinction. Journal of Neuroscience, 39(17), 3264-3276. https://doi.org/10.1523/ JNEUROSCI.2713-18.2019

Ekman, P., \& Friesen, W. V. (1976). Measuring facial movement. Environmental Psychology and Nonverbal Behavior, 1, 56-75. https:// doi.org/10.1007/BF01115465

Freeston, M. H., Rhéaume, J., Letarte, H., Dugas, M. J., \& Ladouceur, R. (1994). Why do people worry? Personality and Individual Differences, 17(6), 791-802. https://doi.org/10.1016/0191-8869(94)90048-5

Graham, B., \& Milad, M. (2011). The study of fear extinction: Implications for anxiety disorders. American Journal of Psychiatry, 168, 1255-1265. https://doi.org/10.1176/appi.ajp.2011.11040557

Grös, D. F., Antony, M. M., Simms, L. J., \& McCabe, R. E. (2007). Psychometric properties of the State-Trait Inventory for Cognitive and Somatic Anxiety (STICSA): Comparison to the State-Trait Anxiety Inventory (STAI). Psychological Assessment, 19(4), 369-381. https://doi.org/10.1037/1040-3590.19.4.369

Grupe, D. W., \& Nitschke, J. B. (2013). Uncertainty and anticipation in anxiety: An integrated neurobiological and psychological perspective. Nature Reviews Neuroscience, 14(7), 488-501. https://doi.org/10.1038/nrn3524

Hofmann, S. G. (2008). Cognitive processes during fear acquisition and extinction in animals and humans: Implications for exposure therapy of anxiety disorders. Clinical Psychology Review, 28(2), 199-210. https://doi.org/10.1016/j.cpr.2007.04.009

Krypotos, A. M., \& Engelhard, I. M. (2018). Testing a novelty-based extinction procedure for the reduction of conditioned avoidance. Journal of Behavior Therapy and Experimental Psychiatry, 60, 22-28. https://doi.org/10.1016/j.jbtep.2018.02.006

Lonsdorf, T. B., Menz, M. M., Andreatta, M., Fullana, M. A., Golkar, A., Haaker, J., Heitland, I., Hermann, A., Kuhn, M., Kruse, O., Meir Drexler, S., Meulders, A., Nees, F., Pittig, A., Richter, J., Römer, S., Shiban, Y., Schmitz, A., Straube, B., ... Merz, C. J. (2017). Don't fear 'fear conditioning': Methodological considerations for the design and analysis of studies on human fear acquisition, extinction, and return of fear. Neuroscience \& Biobehavioral Reviens, 77, 247-285. https://doi. org/10.1016/j.neubiorev.2017.02.026

Lonsdorf, T. B., \& Merz, C. J. (2017). More than just noise: Inter-individual differences in fear acquisition, extinction and return of fear in humans-Biological, experiential, temperamental factors, and methodological pitfalls. Neuroscience \& Biobehavioral Reviews, 80, 703-728. https://doi.org/10.1016/j.neubiorev.2017.07.007

Lonsdorf, T. B., Merz, C. J., \& Fullana, M. A. (2019). Fear extinction retention: Is it what we think it is? Biological Psychiatry, 85(12), 1074-1082. https://doi.org/10.1016/j.biopsych.2019.02.011

Lucas, K., Luck, C. C., \& Lipp, O. V. (2018). Novelty-facilitated extinction and the reinstatement of conditional human fear. Behaviour Research and Therapy, 109, 68-74. https://doi.org/10.1016/j.brat.2018.08.002

Milad, M. R., \& Quirk, G. J. (2012). Fear extinction as a model for translational neuroscience: Ten years of progress. Annual Review of Psychology, 63, 129-151. https://doi.org/10.1146/annurev.psych.121208.131631

Molapour, T., Golkar, A., Navarrete, C. D., Haaker, J., \& Olsson, A. (2015). Neural correlates of biased social fear learning and interaction in an intergroup context. NeuroImage, 121, 171-183. https://doi.org/10.1016/j.neuroimage.2015.07.015

Morriss, J., Chapman, C., Tomlinson, S., \& Van Reekum, C. M. (2018). Escape the bear and fall to the lion: The impact of avoidance availability on threat acquisition and extinction. Biological Psychology, 138, 73-80. https://doi.org/10.1016/j.biopsycho.2018.08.017

Morriss, J., Christakou, A., \& Van Reekum, C. M. (2015). Intolerance of uncertainty predicts fear extinction in amygdala-ventromedial prefrontal cortical circuitry. Biology of Mood \& Anxiety Disorders, 5(1), 1. https://doi.org/10.1186/s13587-015-0019-8

Morriss, J., Christakou, A., \& Van Reekum, C. M. (2016). Nothing is safe: Intolerance of uncertainty is associated with compromised fear extinction learning. Biological Psychology, 121, 187-193. https://doi.org/10.1016/j.biopsycho.2016.05.001

Morriss, J., Macdonald, B., \& van Reekum, C. M. (2016). What is going on around here? Intolerance of uncertainty predicts threat generalization. PLoS One, 11(5), e0154494. https://doi.org/10.1371/journal.pone.0154494

Morriss, J., McSorley, E., \& van Reekum, C. M. (2017). I don't know where to look: The impact of intolerance of uncertainty on saccades towards non-predictive emotional face distractors. Cognition and Emotion, 32(5), 1-10.

Morriss, J., Saldarini, F., Chapman, C., Pollard, M., \& van Reekum, C. M. (2019). Out with the old and in with the new: The role of intolerance of uncertainty in reversal of threat and safety. Journal of Experimental Psychopathology, 10(1), 1-11. https://doi. org $/ 10.1177 / 2043808719834451$

Morriss, J., \& van Reekum, C. M. (2019). I feel safe when I know: Contingency information promotes threat extinction in high intolerance of uncertainty individuals. Behaviour Research and Therapy, 116, 111-118.

Morriss, J., Wake, S., Lindner, M., McSorley, E., \& Dodd, H. (2020). How many times do I need to see to believe? The impact of intolerance of uncertainty and exposure experience on safety-learning and retention in young adults. International Journal of Psychophysiology, 153, 8-17. https://doi.org/10.1016/j.ijpsycho.2020.04.012 
Olsson, A., Ebert, J. P., Banaji, M. R., \& Phelps, E. A. (2005). The role of social groups in the persistence of learned fear. Science, 309(5735), 785-787.

Peugh, J. L. (2010). A practical guide to multilevel modeling. Journal of School Psychology, 48(1), 85-112. https://doi.org/10.1016/j. jsp.2009.09.002

Quené, H., \& Van den Bergh, H. (2004). On multi-level modeling of data from repeated measures designs: A tutorial. Speech Communication, 43(1), 103-121. https://doi.org/10.1016/j.specom.2004.02.004

Ree, M. J., French, D., MacLeod, C., \& Locke, V. (2008). Distinguishing cognitive and somatic dimensions of state and trait anxiety: Development and validation of the State-Trait Inventory for Cognitive and Somatic Anxiety (STICSA). Behavioural and Cognitive Psychotherapy, 36(3), 313-332. https://doi.org/10.1017/S1352465808004232

Snijders, T. A. (2005). Power and sample size in multilevel linear models. Encyclopedia of Statistics in Behavioral Science, 3, 1570-1573.

Wake, S., Morriss, J., Johnstone, T., Van Reekum, C. M., \& Dodd, H. (2021). Intolerance of uncertainty, and not social anxiety, is associated with compromised extinction of social threat. Behaviour Research and Therapy, 139, 103818. https://doi. org $/ 10.1016 /$ j.brat.2021.103818

Wake, S., van Reekum, C. M., Dodd, H., \& Morriss, J. (2020). The impact of intolerance of uncertainty and cognitive behavioural instructions on safety learning. Cognitive Therapy and Research, 44, 931-942. https://doi.org/10.1007/s10608-020-10113-4

Xia, W., Dymond, S., Lloyd, K., \& Vervliet, B. (2017). Partial reinforcement of avoidance and resistance to extinction in humans. Behaviour Research and Therapy, 96, 79-89. https://doi.org/10.1016/j.brat.2017.04.002

\section{SUPPORTING INFORMATION}

Additional supporting information may be found in the online version of the article at the publisher's website.

How to cite this article: Wake, S., Dodd, H., \& Morriss, J. (2021). Intolerance of uncertainty and novelty facilitated extinction: The impact of reinforcement schedule. British Journal of Psychology, 00, 1-17. https://doi.org/10.1111/bjop.12538 